\title{
Tumour-specific methylation of PTPRG intron 1 locus in sporadic and Lynch syndrome colorectal cancer
}

\author{
Eddy HJ van Roon ${ }^{1,2,4}$, Noël FCC de Miranda ${ }^{1}$, Merlijn P van Nieuwenhuizen ${ }^{1}$, Emile J de Meijer ${ }^{2}$, \\ Marjo van Puijenbroek ${ }^{1}$, Pearlly S Yan ${ }^{3}$, Tim H-M Huang ${ }^{3}$, Tom van Wezel ${ }^{1}$, Hans Morreau ${ }^{\star 1}$ and Judith M Boer ${ }^{\star, 2}$
}

DNA methylation is a hallmark in a subset of right-sided colorectal cancers. Methylation-based screening may improve prevention and survival rate for this type of cancer, which is often clinically asymptomatic in the early stages. We aimed to discover prognostic or diagnostic biomarkers for colon cancer by comparing DNA methylation profiles of right-sided colon tumours and paired normal colon mucosa using an $8.5 \mathrm{k} \mathrm{CpG}$ island microarray. We identified a diagnostic CpG-rich region, located in the first intron of the protein-tyrosine phosphatase gamma gene (PTPRG) gene, with altered methylation already in the adenoma stage, that is, before the carcinoma transition. Validation of this region in an additional cohort of 103 sporadic colorectal tumours and 58 paired normal mucosa tissue samples showed $94 \%$ sensitivity and $96 \%$ specificity. Interestingly, comparable results were obtained when screening a cohort of Lynch syndrome-associated cancers. Functional studies showed that PTPRG intron 1 methylation did not directly affect PTPRG expression, however, the methylated region overlapped with a binding site of the insulator protein CTCF. Chromatin immunoprecipitation (ChIP) showed that methylation of the locus was associated with absence of CTCF binding. Methylation-associated changes in CTCF binding to PTPRG intron 1 could have implications on tumour gene expression by enhancer blocking, chromosome loop formation or abrogation of its insulator function. The high sensitivity and specificity for the PTPRG intron 1 methylation in both sporadic and hereditary colon cancers support biomarker potential for early detection of colon cancer.

European Journal of Human Genetics (2011) 19, 307-312; doi:10.1038/ejhg.2010.187; published online 8 December 2010

Keywords: PTPRG; colorectal cancer; CTCF; DNA methylation; Lynch syndrome

\section{INTRODUCTION}

DNA methylation is a common mechanism in colorectal tumourigenesis. $^{1,2}$ Over the last decade, several genome-wide array-based methods have been developed, allowing the discovery of novel tumour-specific methylated loci. Enzymatic (HELP, ${ }^{3}$ MMASS, ${ }^{4}$ differential methylation hybridisation (DMH) ${ }^{5} \mathrm{CHARM}^{6}$ ) and chromatin immunoprecipitation (ChIP) methods $^{7}$ are most commonly used for genome-wide screening of DNA methylation, in combination with $\mathrm{CpG}$ island or promoter microarrays. An alternative genome-wide approach to identify genes silenced by DNA methylation detects expression differences in cell lines treated with DNA demethylating agents. ${ }^{8,9}$ More recently, captured methylated $\mathrm{DNA}^{10}$ and bisulphite-converted reduced representations ${ }^{11}$ are analysed by high-throughput sequencing strategies. The unbiased approaches have indicated that transcription regulation associated with $\mathrm{CpG}$ methylation is not restricted to promoter $\mathrm{CpG}$ islands. ${ }^{12,13}$ Conserved regions up to $2 \mathrm{kbp}$ distant from the promoter, annotated as $\mathrm{CpG}$ island shores, ${ }^{12}$ and promoter $\mathrm{CpG}$ islands of lesser density, annotated as intermediate-CpG islands, ${ }^{13}$ undergo cancer-specific methylation more often than traditional promoter $\mathrm{CpG}$ islands. The methylation status of these regions is strongly related to gene expression and might have been underestimated in previous studies.
The aim of this study was to discover novel tumour-specific DNA methylation markers in right-sided colon cancer. These tumours have a higher frequency of the CpG island methylator phenotype (CIMP). Additionally, right-sided tumours are often clinically asymptomatic at early stages, thus, patients would greatly benefit from a reliable screening method. We employed DMH combined with a $8.5 \mathrm{k} \mathrm{CpG}$ clone library microarray for the initial identification of differential methylation in a cohort of colon cancers. ${ }^{14}$ This library is enriched for CG-rich areas throughout the genome, encompassing promoter $\mathrm{CpG}$ islands, as well as CpG-rich island shores and intermediate-CpG islands. ${ }^{15}$ We report tumour-specific methylation of the first intron of the receptor protein-tyrosine phosphatase gamma gene (PTPRG), in both sporadic and Lynch syndrome colorectal cancers. Additionally, we demonstrate that methylation of this region affects it's binding to the CCCTC-binding factor (zinc-finger protein, CTCF).

\section{MATERIALS AND METHODS}

Tissue

Anonymized samples were obtained from patients who underwent surgery between 1988 and 2006 at the Leiden University Medical Center (Leiden, The Netherlands) or at the Rijnland Hospital (Leiderdorp, The Netherlands). Tumour sections were micro-dissected to minimise normal epithelium and

\footnotetext{
${ }^{1}$ Department of Pathology, Leiden University Medical Center, Leiden, The Netherlands; ${ }^{2}$ Center for Human and Clinical Genetics, Leiden University Medical Center, Leiden, The Netherlands; ${ }^{3}$ Department of Molecular Virology, Immunology, and Medical Genetics, Division of Human Cancer Genetics, Comprehensive Cancer Center, Ohio State University, Columbus, $\mathrm{OH}$, USA

*Correspondence: Dr JM Boer, Center for Human and Clinical Genetics, Leiden University Medical Center, Postal Zone S4-P, P0 Box 9600,2300 RC Leiden, The Netherlands. Tel: +31 71526 9422; Fax: +31 71526 8285; E-mail: J.M.Boer@LUMC.nl and H Morreau, Department of Pathology, Leiden University Medical Center, Postal Zone L1-Q, PO Box 9600, 2300 RC Leiden, The Netherlands. Tel: +31 71526 6630; Fax: +31 71526 6952; E-mail: J.Morreau@LUMC.nl

${ }^{4}$ Current address: Department of Pediatric Oncology and Hematology, Erasmus Medical Center-Sophia Children's Hospital, Rotterdam, The Netherlands.

Received 10 June 2010; revised 27 September 2010; accepted 7 October 2010; published online 8 December 2010
} 
stromal cells. DNA was isolated from fresh-frozen tissue using a previously described method, ${ }^{16}$ and from formalin-fixed paraffin-embedded (FFPE) tissue using the Wizard Genomic DNA Purification kit (Promega, Madison, WI, USA). We used available normal mucosa from the same individuals as control to correct for age-dependent methylation. Age, location and microsatellite instability (MSI) status for the sporadic tumours are listed in Supplementary Table S1, and for the Lynch syndrome-associated tumours in Supplementary Table S2. The colorectal cancer cell lines SW48, RKO, SW480, Caco2, SW837 and LS411 were obtained from the American Type Culture Collection (Manassas, VA, USA). DNA was isolated from these cell lines as described previously. ${ }^{16}$ RNA was isolated using TRIZOL (Invitrogen, Carlsbad, CA, USA) and subsequently purified with Qiagen RNeasy columns combined with the RNase-free DNase kit (Qiagen Sciences, Germantown, MD, USA). The present study was approved by the Medical Ethics committee of the LUMC (protocol P01-019). Cases were analysed following the medical ethical guidelines described in the Code Proper Secondary Use of Human Tissue established by the Dutch Federation of Medical Sciences.

\section{CpG island microarrays}

CpG island clone inserts (8544) were amplified using vector-based primers as described previously. ${ }^{14,17}$ The CpG island clone library was originally generated by the Sanger Centre from affinity-purified in vitro methylated DNA fragments. ${ }^{15}$ Clone sequence information was downloaded from the Toronto Microarray Facility. PCR products were spotted onto CodeLink (GE Healthcare, Munich, Germany) slides using an OmniGrid arrayer (Genomic Solutions, Ann Arbor, MI, USA) at the Leiden Genome Technology Center (www.lgtc.nl) as described. ${ }^{18}$

\section{Differential methylation hybridisation}

DMH was performed according to Yan et al. ${ }^{14}$ Cy5-labeled amplicons, representing methylated DNA fragments derived from tumours and paired normal mucosa samples, were cohybridized to the $\mathrm{CpG}$ island microarrays with a Cy3-labeled common reference amplicon consisting of a pool of DNA from the six colorectal cancer cell lines described above. Detection was done on a G2565BA scanner (Agilent Technologies, Santa Clara, CA, USA) and image analysis using GenePix6.0 (Molecular Devices, Union City, CA, USA). Preprocessing, including normalisation, was performed using the GenePix error model in Rosetta Resolver version 5.0 (Rosetta Biosoftware, Seattle, WA, USA). Microarray data for the tumour and normal samples were compared using an error-weighted ANOVA model and corrected for multiple testing ${ }^{19}$ in Rosetta Resolver. Microarray data are available on Gene Expression Omnibus with accession number GSE21181.
Bisulphite sequence analysis (BSA)

DNA samples (500 ng) were converted using the EZ DNA methylation Gold bisulphite kit (Zymo Research, Orange, CA, USA). Primers (Supplementary Table S3) for BSA of 10 CpGs in the PTPRGint1 locus (Supplementary Figure S1) were designed using MethPrimer. ${ }^{20}$ Amplification was carried out in a DNA Engine Dyad Peltier Thermal Cycler (Bio-Rad, Hercules, CA, USA) using AmpliTaq Gold PCR buffer and enzyme (Applied Biosystems, Foster City, CA, USA). For the direct BSA, PCR products were sequenced using the right primer, resulting in nine interpretable CpGs (CpG2-10). For additional clonal BSA, PTPRGint1 PCR fragments were cloned into TOP10 Escherichia coli bacteria using a TOPO TA cloning kit (Invitrogen). Sequence alignment analysis was performed using ClustalW ${ }^{21}$ and BioEdit. ${ }^{22} \mathrm{CpG}$ dinucleotides in the direct BSA were scored as being methylated when the ratio of the cytosine/thymine peaks was above 0.4 . The BiQ analyzer software was used for visualisation. ${ }^{23}$

\section{MS-MLPA assay}

Custom MS-MLPA probes (Supplementary Table S3) for the PTPRGint1 locus were designed in Primer $3^{24}$ and included the HhaI site in CpG9 (see Figure 1b). As a control, we used a BRCA2 probe set from the SALSA MS-MLPA KIT ME001B Tumour suppressor-1 kit (MRC-Holland, Amsterdam, The Netherlands). Fragment analysis was performed on an ABI 3130 (Applied Biosystems). The MS-MPLA was performed as described using 50 ng of genomic DNA. ${ }^{25}$ A negative, unmethylated control (human semen DNA) and a 100\% methylated DNA control (CpGenome Universal methylated DNA, Chemicon, Millipore, Billerica, MA, USA), were included in every experiment to assess HhaI cleavage and PCR. Fragment analysis was performed in GeneMapper (Applied Biosystems). PTPRGint1 peak heights were normalised by comparison with the $B R C A 2$ peak height from the same run. Subsequently, the PTPRGint1/BRCA2 ratio from the digested reaction was divided by the PTPRGint1/BRCA2 ratio from the undigested reaction resulting in one ratio per sample that represented the fraction of methylated CpG9. In all, 10 independent measurements provided a ratio distribution for unmethylated (mean, 0.108; SD 0.037) and fully methylated (mean 0.833, SD 0.148) control DNA. Samples were typed as being unmethylated or methylated when they were within three standard deviations of the mean of the unmethylated and methylated reference samples, respectively. Samples with ratios in between these standard deviation boundaries were scored as partially methylated. For specificity and sensitivity calculations, partially methylated samples were considered methylated. Determination of specificity between normal and tumour tissue was performed by a $\chi^{2}$-test.
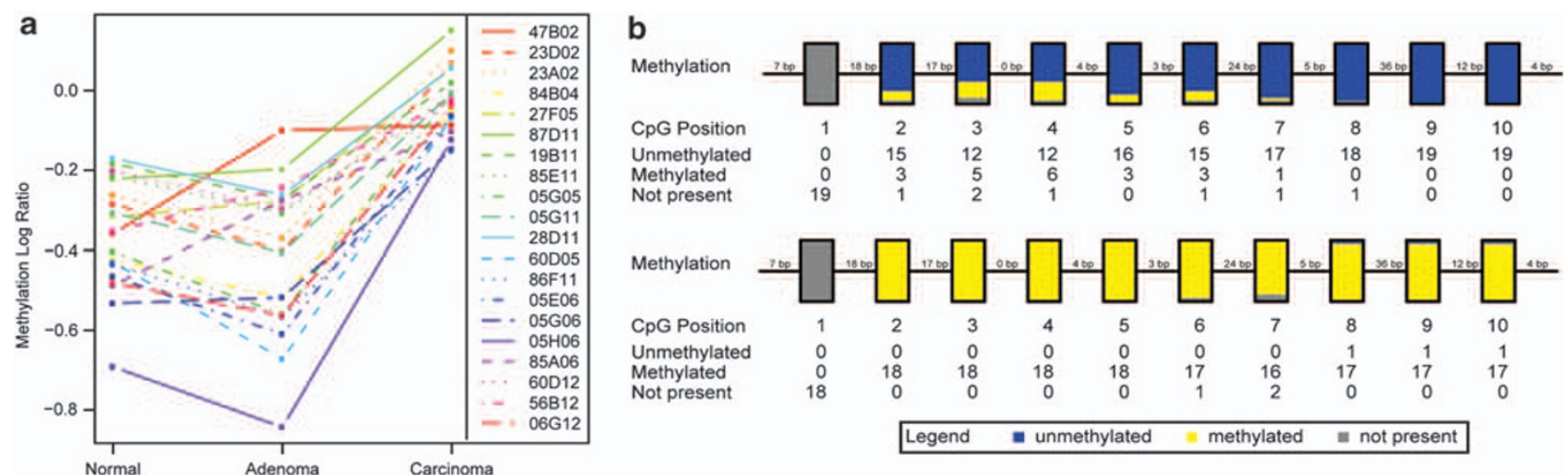

Figure 1 Identification and validation of the differentially methylated locus PTPRGint1. (a) Trend plot of the top-20 differentially methylated microarray clones (FDR $\leq 0.01 \%$ ) showing the average log10 ratios in the normal, adenoma and carcinoma samples compared with the colorectal cancer cell line reference panel. Clone 47B02, corresponding to PTPRGint1, had a similarly increased log ratio in adenomas and carcinomas compared with normals (red solid line). (b) BiQ summary of direct BSA of PTPRGint1 (CpG dinucleotides 1-10) in paired normal colon mucosa ( $n=19$, upper panel) and colon tumours ( $n=18,12$ right- and left-sided carcinomas and six adenomas, lower panel) showed highest specificity and sensitivity in the four most $3^{\prime}$ CpGs measured. Each box corresponds to one $\mathrm{CpG}$ position in the genomic sequence, while colours summarise the methylation states of all sequenced samples at that position. 


\section{Real-time RT-PCR}

cDNA was generated using the random primer protocol from the Transcriptor First Strand cDNA Synthesis Kit (Roche Applied Science, Indianapolis, IN, USA) using $1 \mu \mathrm{g}$ of RNA. Intron-spanning primers (Supplementary Table S3) were designed to target exon 1 and 2 of the main PTPRG transcript in Primer3. ${ }^{24}$ Reactions were performed in duplicate on a Light Cycler 480 (Roche Applied Science) using IQ SYBR Green SuperMix (Biorad). High-resolution melting curve analysis was performed to check primer specificity. Reactions with more than one peak in the melting curve were discarded, as were samples where the standard deviation between technical replicates was above one Ct value. A standard curve was generated using five 1:10 dilutions of pooled cDNA from colon cancer cell lines (SW48, RKO, SW480, Caco2, SW837 and LS411), showing an efficiency of $100 \%$. The Ct values that were used for analysis were between 23 and 33. Relative mRNA concentrations were calculated from this standard curve. Stably expressed control genes for normalisation were selected with the GeNorm applet; the two most stably expressed genes were used for normalisation of each tumour cDNA (CPSF6 and EEF1). ${ }^{26}$

\section{CTCF ChIP and quantitative PCR}

The primary colon cancer cell line KP7038t, established at the Department of Pathology at the LUMC, was grown in GIBCO RPMI 1640 with glutamax (Invitrogen), 10\% fetal calf serum and penicillin/streptomycin $(50 \mu \mathrm{g} / \mathrm{ml})$. Tumour-associated fibroblasts (KP7038f) collected from the primary tumour, as well as cell lines RKO and SW480 were grown under identical conditions. Cells at approximately $80 \%$ confluency were fixed with $1 \%$ formaldehyde for $10 \mathrm{~min}$ at room temperature. The formaldehyde was quenched with glycine $(0.125 \mathrm{M})$ and the cells were harvested by scraping. ChIPs were performed using the SimpleChIP Enzymatic Chromatin IP kit (Cell Signaling Technology, Danvers, MA, USA) with $10 \mu \mathrm{l}$ anti-CTCF antibody (D31H2 XP; Cell Signaling Technology). Normal colon mucosa was collected from a patient who underwent a colon colonoscopy unrelated to colon cancer. Twenty $50 \mu \mathrm{m}$ sections, cut from macro-dissected frozen tissue, were fixed in PBS with $1 \%$ formaldehyde. After quenching, the tissue was micro-dissected and processed for ChIP followed by duplicate PCR reactions as above. Primers (Supplementary Table S3) targeting control regions were selected from Kim et al. ${ }^{27}$ The Ct values that were used for analysis were between 25 and 40. Standard curves were generated using four consecutive 1:5 dilutions of input DNA for both cultures (non-immunoprecipitated, cross-linked DNA) to determine relative DNA concentrations. For comparison between pull-downs, relative DNA concentrations of the CTCF and IgG antibody pull-downs were normalised with the corresponding relative concentration from the histone $\mathrm{H} 3$ antibody pull down.

\section{RESULTS}

Locus PTPRGint1 is methylated in colorectal adenomas and carcinomas

Methylation profile comparison by ANOVA of 15 carcinomas, 3 adenomas and 8 paired normal mucosa samples, identified 20 differentially methylated loci for the three tissue groups (false discovery rate $<0.01 \%$ ). For all but one of these loci, methylation in the adenomas was comparable to the normal samples whereas carcinomas showed increased methylation (Figure 1a). The most significant $\mathrm{CpG}$ island clone was 47B02 that showed increased methylation in both adenomas and carcinomas compared with normal mucosa. Therefore, we performed validation experiments for the corresponding locus, which mapped to the first intron of the PTPRG gene (chr3: 61524993-61525363, UCSC assembly: March 2006, see Supplementary Figure S1), referred to as PTPRGint1.

Analysis at single $\mathrm{CpG}$ resolution using direct BSA showed that 17 out of 18 tumour samples were fully methylated in the PTPRGint1 region, whereas one carcinoma was unmethylated for CpG dinucleotides 8-10 (Figure 1b, bottom panel). In contrast, normal colon samples were mostly unmethylated (Figure $1 \mathrm{~b}$, upper panel). CpGs 7-10 were most informative to distinguish between tumour and normal in this set of samples. These results were confirmed at the single chromosome level using clonal BSA (Supplementary Figure S2). In summary, the microarray-based finding of differential PTPRGint1 methylation in right-sided tumours was confirmed, and extended to left-sided adenomas and carcinomas.

Methylation of PTPRGint1 CpG9 has high sensitivity and specificity To assess the sensitivity and specificity of PTPRGint 1 methylation to discriminate between cancer and normal tissue, we developed a highthroughput MS-MLPA assay (Figures 2a and b). We tested an FFPE cohort of 103 tumours and 58 corresponding normal tissues, which allowed us to assess the possibility of age-related methylation often seen in aging mucosa. Of the 67 carcinoma samples, 94\% showed methylation of the targeted CpG dinucleotide (61 fully, two partially methylated), whereas 46 (95.8\%) of the 48 corresponding normal samples were unmethylated, and the remaining two partially methylated (Figure $2 \mathrm{c}$ and Table 1). Comparing the methylation numbers between normal and tumour tissue by $\chi^{2}$-test provided a highly significant $P$-value of $9.8 \times 10^{-110}$. PTPRGintl methylation was independent of MSI status in the sporadic carcinomas, as both MSI-High and stable cases were methylated. A relatively small group of 18 sporadic adenomas (13 low grade dysplastic and five high grade dysplastic) and neighbouring normal mucosa of 10 of these showed PTPRGint1 methylation in all adenomas, but not in the available normal mucosa (Figure 2c and Table 1). To assess whether PTPRGint1 methylation is an early event in colorectal carcinogenesis, several colon lesions preceding the adenoma/carcinoma stages were studied. All six hyperplastic polyps and 11 out of 12 serrated adenomas tested showed PTPRGint1 methylation (Figure 2c).

\section{PTPRGint1 CpG9 methylation in Lynch syndrome-associated colorectal cancer}

The initial cohort for MS-MLPA validation contained two Lynch syndrome-associated colorectal carcinomas that were both methylated. Therefore, we further investigated PTPRGintl methylation in 63 carcinomas from patients with a DNA mismatch repair (MMR) gene mutation (14 MLH1 mutations, $19 \mathrm{MSH} 2$ mutations and 30 MSH6 mutations). Of these, $92.1 \%$ showed methylation (58 fully, 3 partially methylated), while 62 of the $65(95.7 \%)$ corresponding normal samples were unmethylated (Figure 2c, Table $1 ; \chi^{2} P$-value $3.3 \times 10^{-153}$ ). In conclusion, methylation of PTPRGint1 CpG9 has similarly high sensitivity and specificity in Lynch syndrome-associated colorectal carcinomas as in sporadic colorectal cancer.

\section{No silencing of PTPRG gene expression in methylated samples}

To assess whether hypermethylation of PTPRGint1 affected expression of the gene, relative mRNA levels of PTPRG were studied in 15 right-sided colon carcinomas, 3 adenomas and 18 corresponding normal mucosa samples. The PTPRG gene encodes four protein coding isoforms (Source: HGNC Symbol; Acc:9671, aligned to Ensembl GRCh37). Using intron 1 spanning primers the full length transcripts ENST00000474889 and ENST00000295874 (missing one cassette exon), were analysed (Figure 3 ). Two additional transcripts, ENST00000383711 and ENST00000394462, both starting at exon 3, were analysed using intron 26 spanning primers and gave comparable results (data not shown). Expression of the PTPRG gene was detected in all samples. To assess the effects of PTPRGintl methylation on PTPRG expression, we compared the MS-MLPA methylation ratio with the PTPRG relative expression (Figure 3). We found that PTPRG was expressed at similar levels in the tumour and normal samples independent of methylation status. One patient (No. 28) showed increased PTPRG expression in both normal and tumour samples, 


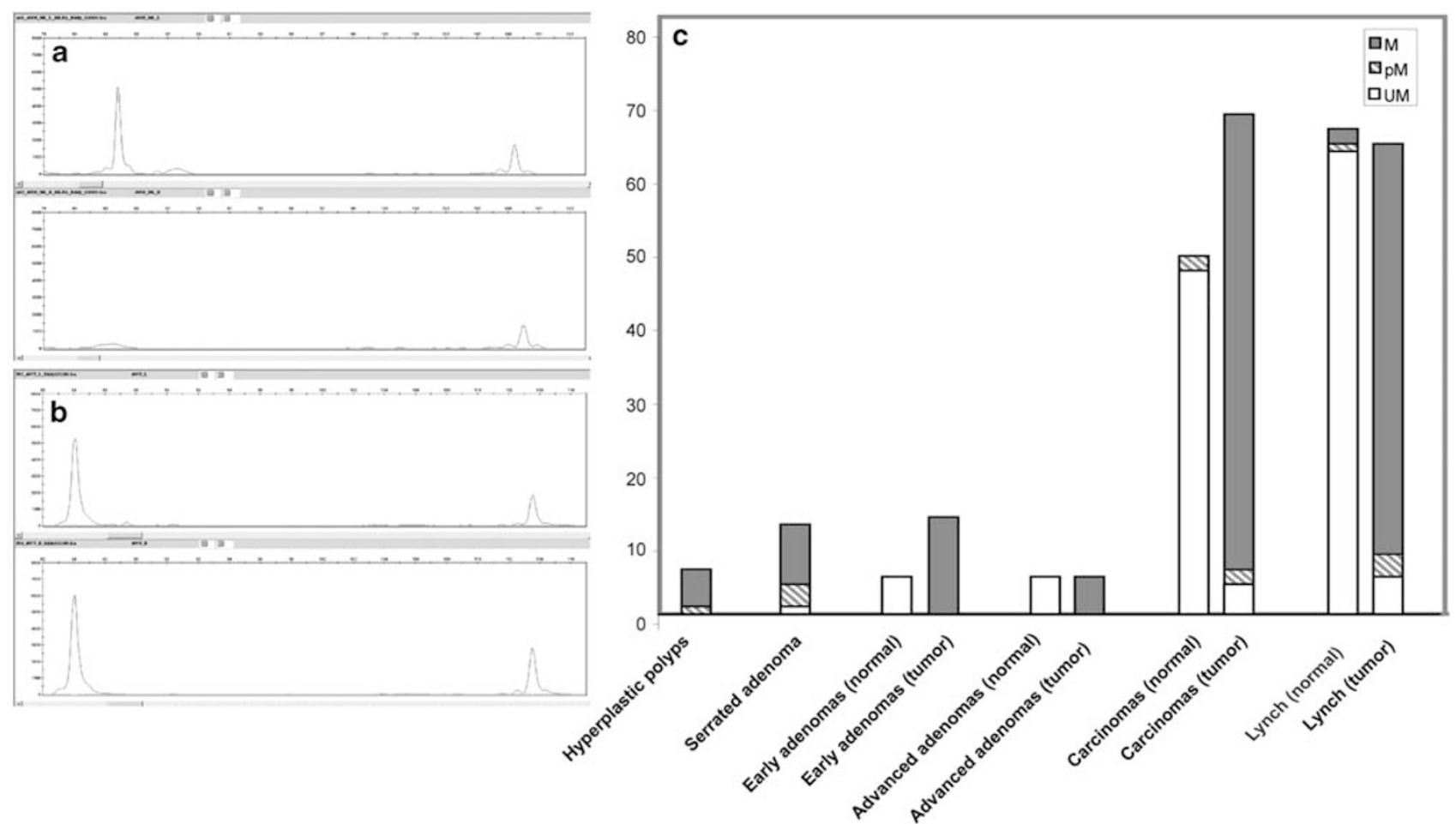

Figure 2 PTPRGint1 methylation detected by MS-MLPA. (a) GeneMapper output of the custom MS-MLPA to analyse PTPRGint1 CpG9 methylation in a normal mucosa sample. Upper panel: The PTPRGint1 peak was located at $\sim 84 \mathrm{bp}$, the control peak was located at $\sim 110 \mathrm{bp}$. Lower panel: Loss of PTPRGint1 signal after Hhal digestion indicated an unmethylated CpG9. (b) GeneMapper output of the custom PTPRGint1 MS-MLPA in the corresponding colon cancer sample. Upper panel: Undigested. Lower panel: Hhal digestion. Retention of the PTPRGint1 marker signal indicated protection against Hhal digestion by CpG9 methylation. (c) Frequency of PTPRGint1 CpG9 methylation in precursor lesions (hyperplastic polyps and serrated adenoma), early- and advanced adenomas, carcinomas and corresponding normal mucosal tissue for the latter three groups. The number of samples typed as methylated (dark), partially methylated (striped) and unmethylated (white) in the MS-MLPA assay is indicated on the $y$ axis.

Table 1 Sensitivity and specificity of the PTPRGint1 locus CpG9 methylation in sporadic tumours and tumours associated with a specific MMR mutation

\begin{tabular}{lcc}
\hline & $\begin{array}{c}\text { Sensitivity } \\
\text { CpG9 }\end{array}$ & $\begin{array}{c}\text { Specificity unmethylated } \\
\text { nothylated tumours/total tumours }\end{array}$ \\
\hline Sporadic adenomas & $100 \%(18 / 18)$ & $100 \%(10 / 10)$ \\
Sporadic carcinomas & $94 \%(63 / 67)$ & $95.8 \%(46 / 48)$ \\
MLH1 mutated & $100 \%(14 / 14)$ & $87 \%(20 / 23)$ \\
MSH2 mutated & $96 \%(18 / 19)$ & $100 \%(24 / 24)$ \\
MSH6 mutated & $86.7 \%(26 / 30)$ & $100 \%(14 / 14)$ \\
Total Lynch & $92.1 \%(58 / 63)$ & $95.4 \%(62 / 65)$
\end{tabular}

Abbreviation: PTPRGint1, protein-tyrosine phosphatase gamma gene intron 1.

aThe mutational analysis of the MMR genes was incomplete for four unpaired normal samples in the Lynch syndrome cohort. Therefore, these were only included in the total specificity calculations.

thought to reflect individual expression differences and a possible copy number effect in the tumour.

\section{PTPRGint1 is a methylation-dependent CTCF binding site}

The PTPRGintl locus overlapped with an experimentally defined CTCF binding site from a CTCF ChIP-chip study in fibroblasts ${ }^{27}$ displayed in the genome web browsers USCS ${ }^{28}$ and Ensembl. ${ }^{29}$ This CTCF binding region of $750 \mathrm{bp}$ (OREG0015647; chr3: 6152510161525851, UCSC assembly: March 2006) has a 262 bp overlap with the $3^{\prime}$ part of the PTPRGint1 locus ( Supplementary Figure S1). We studied CTCF binding by ChIP in the primary tumour culture

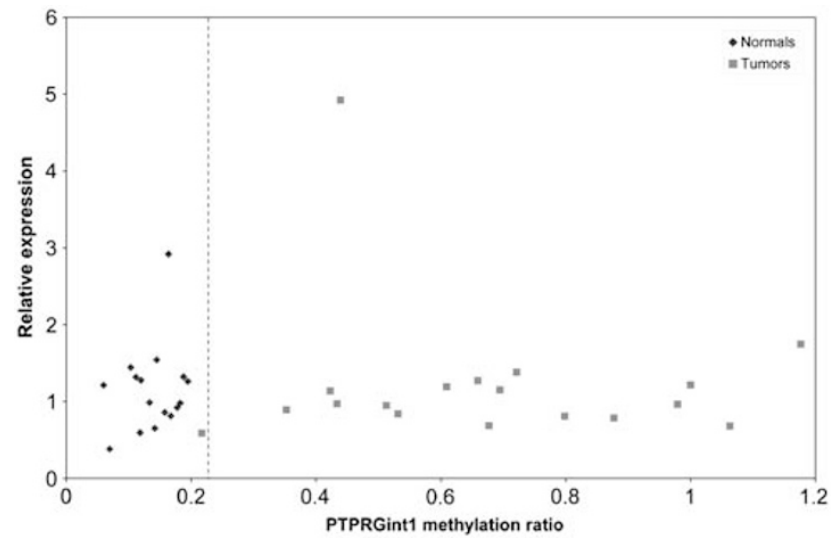

Figure 3 Scatter plot of the relative PTPRG expression against the PTPRGint1 methylation ratio according to the MS-MLPA. The vertical line at 0.22 indicates the cut-off for unmethylated samples. Plotted data is representative of two independent experiments.

KP7038t that carried PTPRGint1 methylation (MS-MLPA ratio 0.97; data not shown), unmethylated tumour-derived fibroblasts KP7038f from the same patient (MS-MLPA ratio 0.07, data not shown), as well as normal colon mucosa. We detected CTCF binding to PTPRGint1 in the normal mucosa and KP7038f fibroblasts, but little binding to the primary tumour cells (Figure $4 \mathrm{a}$ ). 

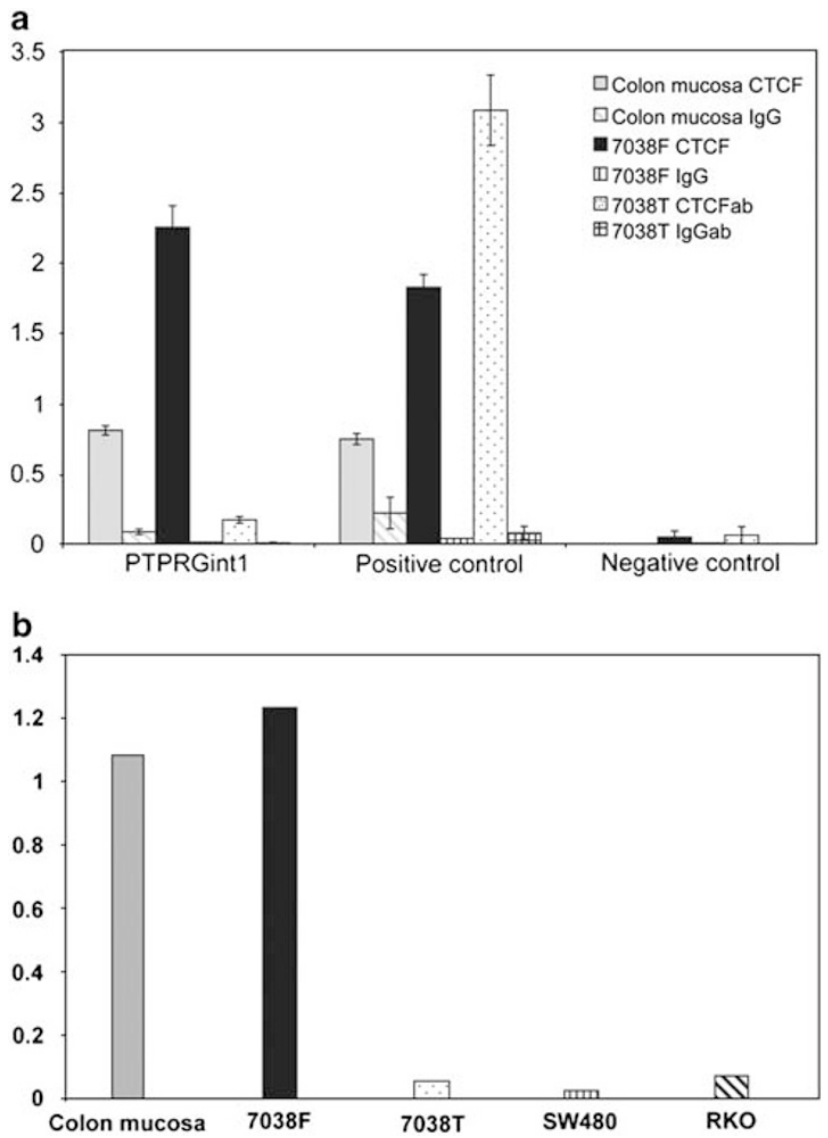

Figure 4 (a) CTCF binding to PTPRGint1, positive- and negative control regions in normal colon mucosa (light grey), KP7038f (black) and KP7038t (dotted). The histone H3 normalised values of the CTCF antibody and IgG negative control antibody pull downs are given per primer pair. Standard errors represent the variability of duplicate PCR reactions. This is a representative experiment of three ChIPs. (b) The PTPRGint1/positive control ratio for normal colon mucosa, KP7038f, KP7038t (also shown in a), SW480 (vertically striped) and RKO (diagonally striped).

To control for possible differences in the amount of CTCF protein between the samples, we compared the qPCR results for the PTPRGint1 region with a positive control locus 7.9 Mb distant from PTPRGintl that was shown to bind CTCF and does not contain a CpG in its putative $20 \mathrm{bp}$ consensus sequence. ${ }^{27}$ This locus was enriched in pull downs of all samples, including the primary tumour cells, indicating that lack of CTCF binding to PTPRGintl was not because of lack of CTCF protein. CTCF binding to PTPRGint1 was comparable to the positive control in both KP7038f fibroblasts (ratio 1.2) and normal colon mucosa (ratio 1.1). However, the PTPRGint1/ positive control ratio was 0.06 in the primary tumour culture KP7038t (Figure 4b). CTCF binding to PTPRGintl was similarly decreased in colorectal cancer cell lines RKO and SW480 (Figure 4b). These results indicate a significant decrease of bound CTCF to the methylated PTPRGint1 region in tumour cells.

\section{DISCUSSION}

We describe colorectal tumour-specific methylation of a locus, in the first intron of the putative tumour suppressor gene PTPRG, in both proximal and distal carcinomas and adenomas, including Lynch syndrome tumours. For these high-risk individuals, who are advised to undergo regular colonoscopies, no molecular markers have been described so far. ${ }^{30}$ Assuming that a successful faecal or blood DNA test for PTPRGintl methylation could be developed, this is a promising discovery that would aid the early detection of colorectal tumours, independent of their aetiology.

PTPRGintl is not located in a promoter CpG island but in the first intron, about $3 \mathrm{kbp}$ from the transcriptional start site. We did not find a relation between the methylation status of PTPRGint1 and PTPRG expression, indicating that PTPRGintl methylation does not lead to loss of function of PTPRG as has been observed for mutations in colon cancer, and deletions in lung carcinomas and renal carcinoma cell lines. ${ }^{31,32}$ However, the identification of a methylation-sensitive CTCF binding site overlapping with PTPRGint1 suggests that tumourspecific methylation may have a more distant effect. Differential binding of the insulator protein CTCF could have a major influence on expression of distant genes through alternative loop formation, as has been observed in $\beta$-globin expression in mouse models. ${ }^{33} \mathrm{~A}$ recent study has shown that loss of CTCF binding to a boundary region upstream of CDKN2A resulted in spreading of repressed chromatin and DNA methylation into the p16 promoter with sequential downregulation of $p 16$ expression. ${ }^{34}$ The same study described that loss of upstream CTCF binding resulted in promoter DNA methylation of RASSF1 and CDH1., ${ }^{34}$ Contradictory to the finding that CTCF binding abrogation was shown to be causative of heterochromatin spreading and DNA methylation ${ }^{34,35}$ is the observation that DNA methylation of CTCF binding sites is suggested to regulate CTCF binding. ${ }^{36,37}$ Interestingly, aberrant DNA methylation that excludes CTCF binding to intronic regulatory DNA was shown to promote expression of an oncogene, BCL6, in B cell lymphomas. ${ }^{38}$ Although the sequence of events is unknown, the age-related aberrant hypermethylation often seen in colon cancer hints towards the latter.

We excluded differential peptidic abundance of CTCF between tumour and normal samples by successfully amplifying a positive control CTCF binding site on all samples. It remains to be demonstrated whether CTCF protein modifications and its cellular and nuclear distribution are maintained in all tumour cells, both of which can influence CTCF activity and the binding to specific regions. More insight into the role of aberrant DNA methylation of PTPRGintl in the aetiology of cancer requires a better understanding of whether aberrant CTCF binding is caused by inhibition of protein activity or by initial aberrant methylation of the CTCF binding site.

Hypermethylation of the CpG island in the PTPRG gene promoter has been previously described in cutaneous T-cell lymphomas, melanoma cell lines and gastric cancer. ${ }^{39-41}$ Transcriptional downregulation was shown to be associated with $P T P R G$ promoter methylation in the cutaneous T-cell lymphomas study. ${ }^{39}$ This study used a similar microarray for identification of differential methylation as the present study. We did not find differential methylation of the PTPRG promoter region between normal and colon tumour samples on the microarray. Moreover, BSA of colorectal cancer cell lines showed that the PTPRG promoter region was unmethylated ( $\mathrm{R}$ van Doorn, personal communication). Therefore, we have no indication for upstream spreading of DNA methylation from the CTCF binding region at PTPRGintl towards the promoter.

In conclusion, this study provided evidence for tumour-specific hypermethylation of a CTCF binding site located in the first intron of PTPRG. The high specificity and sensitivity imply a possible utility for PTPRGint1 methylation in new or existing colon-specific methylation marker panels. Especially, the high level of PTPRGintl methylation in Lynch syndrome-associated colorectal tumours is unique and could prove to be a valuable addition. Methylation-dependent absence of CTCF binding to the PTPRGintl locus suggests a possible effect on 
chromatin density or conformation that could have a role in colon tumourigenesis. ${ }^{42}$

\section{CONFLICT OF INTEREST}

The authors (EHJvR, HM and JB) have filed a patent application pertaining to intellectual property related in part to this report (P78692EP00 Submitted December 31, 2007). All other authors have nothing to disclose.

\section{ACKNOWLEDGEMENTS}

The authors thank Remco van Doorn and Wim Zoutman for sharing protocols and unpublished data and for technical assistance with the library amplification. The authors would also like to thank Jaap van Eendenburg for establishing primary culture KP7038. This study was supported by grants from the Stichting professor AAH Kassenaar fund and the Centre for Medical Systems Biology within the framework of the Netherlands Genomics Initiative (NGI)/Netherlands Organization for Scientific Research (NWO).

1 Grady WM, Carethers JM: Genomic and epigenetic instability in colorectal cancer pathogenesis. Gastroenterology 2008; 135: 1079-1099.

2 Hiltunen MO, Alhonen L, Koistinaho $\mathrm{J}$ et al: Hypermethylation of the APC (adenomatous polyposis coli) gene promoter region in human colorectal carcinoma. Int J Cancer 1997; 70: 644-648.

3 Khulan B, Thompson RF, Ye K et al: Comparative isoschizomer profiling of cytosine methylation: the HELP assay. Genome Res 2006; 16: 1046-1055.

4 Ibrahim AE, Thorne NP, Baird K et al: MMASS: an optimized array-based method for assessing CpG island methylation. Nucleic Acids Res 2006; 34: e136.

5 Huang TH, Perry MR, Laux DE: Methylation profiling of CpG islands in human breast cancer cells. Hum Mol Genet 1999; 8: 459-470.

6 Irizarry RA, Ladd-Acosta C, Carvalho B et al: Comprehensive high-throughput arrays for relative methylation (CHARM). Genome Res 2008; 18: 780-790.

7 Weber M, Davies JJ, Wittig D et al: Chromosome-wide and promoter-specific analyses identify sites of differential DNA methylation in normal and transformed human cells. Nat Genet 2005; 37: 853-862.

8 Schuebel KE, Chen W, Cope L et al: Comparing the DNA hypermethylome with gene mutations in human colorectal cancer. PLoS Genet 2007; 3: 1709-1723.

9 Suzuki $H$, Gabrielson E, Chen W et al: A genomic screen for genes upregulated by demethylation and histone deacetylase inhibition in human colorectal cancer. Nat Genet 2002; 31: 141-149.

10 Down TA, Rakyan VK, Turner DJ et al: A Bayesian deconvolution strategy for immunoprecipitation-based DNA methylome analysis. Nat Biotechnol 2008; 26: 779-785.

11 Meissner A, Mikkelsen TS, Gu H et al: Genome-scale DNA methylation maps of pluripotent and differentiated cells. Nature 2008; 454: 766-770.

12 Irizarry RA, Ladd-Acosta C, Wen B et al: The human colon cancer methylome shows similar hypo- and hypermethylation at conserved tissue-specific $\mathrm{CpG}$ island shores. Nat Genet 2009; 41: 178-186.

13 Weber M, Hellmann I, Stadler MB et al: Distribution, silencing potential and evolutionary impact of promoter DNA methylation in the human genome. Nat Genet 2007; 39: 457-466.

14 Yan PS, Chen CM, Shi $\mathrm{H}$ et al: Dissecting complex epigenetic alterations in breast cancer using CpG island microarrays. Cancer Res 2001; 61: 8375-8380.

15 Cross SH, Charlton JA, Nan X et al: Purification of CpG islands using a methylated DNA binding column. Nat Genet 1994; 6: 236-244.
16 Isola J, DeVries S, Chu L et al: Analysis of changes in DNA sequence copy number by comparative genomic hybridization in archival paraffin-embedded tumor samples. Am J Pathol 1994; 145: 1301-1308.

17 Yan PS, Efferth T, Chen HL et al: Use of CpG island microarrays to identify colorectal tumors with a high degree of concurrent methylation. Methods 2002; 27: 162-169.

18 Knijnenburg J, Szuhai K, Giltay J et al: Insights from genomic microarrays into structural chromosome rearrangements. Am J Med Genet A 2005; 132: 36-40.

19 Benjamini $Y$, Hochberg Y: Controlling the false discovery rate - a practical and powerful approach to multiple testing. J R Stat Soc S B-Methodol 1995; 57: 289-300.

20 Li LC, Dahiya R: MethPrimer: designing primers for methylation PCRs. Bioinformatics 2002; 18: 1427-1431.

21 Larkin MA, Blackshields G, Brown NP et al: Clustal W and Clustal X version 2.0. Bioinformatics 2007; 23: 2947-2948.

22 Tom Hall. BioEdit (URL: http://www.mbio.ncsu.edu/BioEdit/). 2008.

23 Bock C, Reither S, Mikeska T et al: BiQ Analyzer: visualization and quality control for DNA methylation data from bisulfite sequencing. Bioinformatics 2005; 21 : 4067-4068.

24 Rozen S, Skaletsky H: Primer3 on the WWW for general users and for biologist programmers. Methods Mol Biol 2000; 132: 365-386.

25 Nygren AO, Ameziane N, Duarte HM et al: Methylation-specific MLPA (MS-MLPA): simultaneous detection of CpG methylation and copy number changes of up to 40 sequences. Nucleic Acids Res 2005; 33: e128.

26 Vandesompele J, De PK, Pattyn F et al: Accurate normalization of real-time quantitative RT-PCR data by geometric averaging of multiple internal control genes. Genome Biol 2002; 3: RESEARCH0034.

$27 \mathrm{Kim} \mathrm{TH}$, Abdullaev ZK, Smith AD et al: Analysis of the vertebrate insulator protein CTCF-binding sites in the human genome. Cell 2007; 128: 1231-1245.

28 Kuhn RM, Karolchik D, Zweig AS et al: The UCSC Genome Browser Database: update 2009. Nucleic Acids Res 2009; 37: D755-D761.

29 Hubbard TJ, Aken BL, Ayling S et al: Ensembl 2009. Nucleic Acids Res 2009; 37: D690-D697.

30 Alemayehu A, Sebova K, Fridrichova I: Redundant DNA methylation in colorectal cancers of Lynch-syndrome patients. Genes Chromosomes Cancer 2008; 47: 906-914.

31 LaForgia S, Morse B, Levy J et al: Receptor protein-tyrosine phosphatase gamma is a candidate tumor suppressor gene at human chromosome region 3p21. Proc Natl Acad Sci U S A 1991; 88: 5036-5040.

32 Wang Z, Shen D, Parsons DW et al: Mutational analysis of the tyrosine phosphatome in colorectal cancers. Science 2004; 304: 1164-1166.

33 Hou C, Zhao H, Tanimoto K et al: CTCF-dependent enhancer-blocking by alternative chromatin loop formation. Proc Natl Acad Sci USA 2008; 105: 20398-20403.

34 Witcher M, Emerson BM: Epigenetic silencing of the p16(INK4a) tumor suppressor is associated with loss of CTCF binding and a chromatin boundary. Mol Cell 2009; 34: 271-284.

35 Tiwari VK, Baylin SB: Breaching the boundaries that safeguard against repression. Mol Cell 2009; 34: 395-397.

36 De La Rosa-Velazquez IA, Rincon-Arano H, itez-Bribiesca L et al: Epigenetic regulation of the human retinoblastoma tumor suppressor gene promoter by CTCF. Cancer Res 2007; 67: 2577-2585.

$37 \mathrm{Xu} \mathrm{J}$, Huo D, Chen Y et al: CpG island methylation affects accessibility of the proximal BRCA1 promoter to transcription factors. Breast Cancer Res Treat 2009.

38 Lai AY, Fatemi M, Dhasarathy A et al: DNA methylation prevents CTCF-mediated silencing of the oncogene BCL6 in B cell lymphomas. J Exp Med 2010; 207: 1939-1950.

39 van Doorn R, Zoutman WH, Dijkman R et al: Epigenetic profiling of cutaneous T-cell lymphoma: promoter hypermethylation of multiple tumor suppressor genes including BCL7a, PTPRG, and p73. J Clin Oncol 2005; 23: 3886-3896.

40 Furuta J, Nobeyama $Y$, Umebayashi $Y$ et al: Silencing of Peroxiredoxin 2 and aberrant methylation of $33 \mathrm{CpG}$ islands in putative promoter regions in human malignant melanomas. Cancer Res 2006; 66: 6080-6086.

41 Wang JF, Dai DQ: Metastatic suppressor genes inactivated by aberrant methylation in gastric cancer. World J Gastroenterol 2007; 13: 5692-5698.

42 Lewis A, Murrell A: Genomic imprinting: CTCF protects the boundaries. Curr Biol 2004; 14: R284-R286.

Supplementary Information accompanies the paper on European Journal of Human Genetics website (http://www.nature.com/ejhg) 